\title{
Cross-Phase Modulation in a Hydrogenated Amorphous Silicon Optical Fiber
}

\author{
P. Mehta ${ }^{1 *}$, N. Healy ${ }^{1}$, T.D. Day ${ }^{2}$, P.J.A. Sazio ${ }^{1}$, J.V. Badding ${ }^{2}$, and A.C. Peacock ${ }^{1}$ \\ ${ }^{1}$ Optoelectronics Research Centre, University of Southampton, Southampton SO17 1BJ, UK \\ ${ }^{2}$ Department of Chemistry and Materials Research Institute, Pennsylvania State University 16802 PA, USA \\ *pm4g09@orc.soton.ac.uk
}

\begin{abstract}
We experimentally demonstrate cross-phase modulation (XPM) in a hydrogenated amorphous silicon-silica optical fiber. Additional numerical analysis shows that shifts in the probe wavelength are induced by the pump indicating potential for Kerr based switching applications.
\end{abstract}

(C) 2012 Optical Society of America

OCIS codes: (060.2270) Fiber characterization; (060.2290) Fiber materials; (060.4370) Nonlinear optics, fibers;

\section{Introduction}

Optical fibers with novel functionality are promising candidates for a variety of potential applications, such as alloptical signal processors, in transmission windows beyond that of conventional fibers. In particular, hydrogenated amorphous silicon-core (a-Si:H) optical fibers have demonstrated strong optical nonlinearity in short centimeter fiber lengths [1]. A number of nonlinear mechanisms have been observed and characterized in these fibers such as twophoton absorption (TPA), free-carrier absorption (FCA), self-phase modulation (SPM) and cross-absorption modulation (XAM). In order to observe cross-phase modulation (XPM) effects from a high power source on a separate weak signal, we employ a pump-probe technique to establish the extent of nonlinear frequency modulation and shifting caused by XPM [2].

\section{Theory and Experiment}

Within a semiconductor optical fiber, the propagation of a strong pump field $\left(A_{p}\right)$ in the presence of a weaker probe signal $\left(A_{s}\right)$ can induce phase coupling between the two fields. The dynamics of this behavior can be modeled by a system of coupled nonlinear differential equations [3]:

$$
\begin{gathered}
\frac{\partial A_{p}}{\partial z}+\beta_{1 p} \frac{\partial A_{p}}{\partial t}+i \frac{\beta_{2 p}}{2} \frac{\partial^{2} A_{p}}{\partial t^{2}}=i A_{p} \gamma_{p}\left(\left|A_{p}\right|^{2}+2\left|A_{s}\right|^{2}\right)-\frac{1}{2}\left(\alpha_{p}+\sigma_{f p}\right) A_{p} \\
\frac{\partial A_{s}}{\partial z}+\beta_{1 s} \frac{\partial A_{s}}{\partial t}+i \frac{\beta_{2 s}}{2} \frac{\partial^{2} A_{s}}{\partial t^{2}}=i A_{s} \gamma_{s}\left(\left|A_{s}\right|^{2}+2\left|A_{p}\right|^{2}\right)-\frac{1}{2}\left(\alpha_{s}+\sigma_{f s}\right) A_{s} \\
\frac{\partial N}{\partial t}=\frac{\beta_{\mathrm{TPA}}}{2 h A_{\text {eff }}^{2}}\left[\frac{1}{v_{p}}\left|A_{p}\right|^{4}+\frac{1}{v_{s}}\left|A_{s}\right|^{4}+\frac{4}{v_{p}}\left|A_{p}\right|^{2}\left|A_{s}\right|^{2}\right]-\frac{N}{\tau} .
\end{gathered}
$$

Here $A_{i}$ is the slowly varying amplitude of the pulse envelopes $(i=p, s), \beta_{1 i}$ and $\beta_{2 i}$ are the first and second-order dispersion parameters, and the complex nonlinear parameter is defined as $\gamma_{i}=\frac{2 \pi n_{2}}{\lambda_{i} A_{\text {eff }}}+i \frac{\beta_{\text {TPA }}}{2 A_{\text {eff }}}$ with the Kerr $\left(n_{2}\right)$ and TPA $\left(\beta_{\text {TPA }}\right)$ coefficients. The final terms represent the linear losses $\alpha_{i}$, and free-carrier contribution by the pump $\sigma_{f i}=$ $\sigma_{i}(1+i \mu) N$ where $\sigma_{i}$ is the free-carrier absorption (FCA) coefficient, $\mu$ the free-carrier dispersion (FCD) parameter, and $N$ the free-carrier density determined by Eq. (3). The material dependent parameters such as $n_{2}, \beta_{\mathrm{TPA}}, \sigma_{\mathrm{FCA}}, \alpha_{i}$ and the free-carrier lifetime $\tau$ have been established in previous studies of a-Si:H fibers [1,4]. Experimental investigations are performed to demonstrate the strength of spectral modulation due to XPM providing the maximum wavelength shift. Eqs. (1)-(3) are used to establish the theoretical maximum center wavelength shift that a probe field experiences in the presence of a strong pump.

The experimental setup is illustrated in Fig. 1(a). A femtosecond pump laser at 1540nm is split into two beams, one directed to the sample, and the other used to create a broadband supercontinuum in 500m of highly nonlinear fiber 


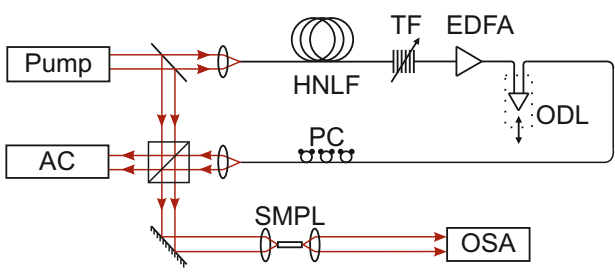

(a)

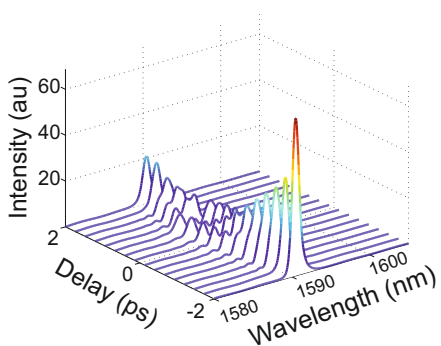

(b)

Fig. 1: (a) Pump-probe experiment for observation of XPM. (b) Experimental results for a 1590nm probe signal experiencing XPM.

(HNLF). The HNLF output is filtered by a tunable filter (TF) to generate a pulse at a different wavelength and finally amplified by an erbium-doped fiber amplifier (EDFA) producing picosecond pulses. The probe's overlap with the pump is controlled by an optical delay line (ODL) and a fiber polarization controller (PC) is used to align the polarization states. An autocorrelator (AC) is used to view the pump-probe delay at one output of the central beam splitter, while the other is focussed into a $1 \mathrm{~cm}$ long $6 \mu \mathrm{m}$ core a-Si:H sample (SMPL) and finally terminated in an optical spectrum analyzer (OSA). Results of XPM at a probe wavelength of $1590 \mathrm{~nm}$ indicates strong spectral modulation as can be seen in Fig. 1(b). The experimental probe spectrum of zero delay is plotted in Fig. 2(a) together with the numerical fit using Eqs. (1)-(3) showing excellent agreement. Furthermore, this analysis may be extended to quantify the shift in the central probe wavelength as a function of delay providing insight for wavelength switching applications. Fig. 2(b) shows the shift in the central wavelength of the probe as a function of delay exhibiting a maximum displacement of $\sim 1 \mathrm{~nm}$. Emphasis on improving the peak wavelength shift for Kerr-induced switching and modulation with maximum probe extinction will be presented.

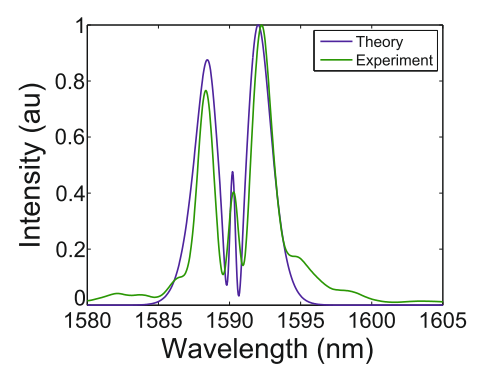

(a)

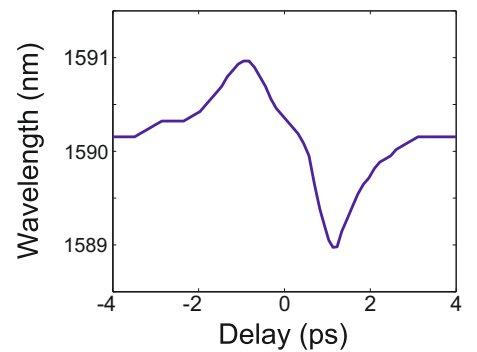

(b)

Fig. 2: XPM at zero delay on the probe signal at $1590 \mathrm{~nm}$. (b) Numerically determined wavelength shift.

\section{Conclusion}

A first demonstration of XPM is observed in an a-Si:H optical fiber. Experimental evidence suggests that Kerr induced spectral modulation manifests as shifts in the central probe wavelength, results of which are accompanied and analyzed with numerical investigations.

\section{References}

1. P. Mehta, N. Healy, N. F. Baril, P. J. A. Sazio, J. V. Badding, and A. C. Peacock, "Nonlinear transmission properties of hydrogenated amorphous silicon core optical fibers," Opt. Express 16, 16826-16831 (2010)

2. I. Hsieh, X. Chen, J. I. Dadap, N. C. Panoiu, and R. M. Osgood, "Cross-phase modulation-induced spectral and temporal effects on copropagating femtosecond pulses in silicon photonic wires," Opt. Express 15, 1135-1146 (2007)

3. T. D. Vo, B. Corcoran, J. Schröder, M. D. Pelusi, D. Xu, A. Densmore, R. Ma, S. Janz, D. J. Moss, and B. J. Eggleton, "Silicon-chip-based real-time dispersion monitoring for 640 Gbit/s DPSK signals," J. Lightwave Tech 29, 1790-1796 (2011)

4. P. Mehta, N. Healy, T. D. Day, J. R. Sparks, P. J. A. Sazio, J. V. Badding, and A. C. Peacock, "All-optical modulation using two-photon absorption in silicon core optical fibers," Opt. Express 19, 19078-19083 (2011) 